\title{
Akustische und optische Echtzeitüberwachung von Bohrprozessen mit passiv-gütegeschaltetem Nd:YAG Laser
}

\author{
Dominik Wild, Peter Kaul und Gerhard Holl \\ Institut für Detektionstechnologien, Institut für Sicherheitsforschung, \\ Hochschule Bonn-Rhein-Sieg, Rheinbach, Deutschland \\ Kontakt: dominik.wild@h-brs.de
}

\begin{abstract}
Zusammenfassung:
In der vorliegenden Arbeit wird ein neuartiges Verfahren zur Echtzeitüberwachung von Laserbohrprozessen vorgestellt. Die Untersuchungen werden an unterschiedlichen Materialien unter Einsatz eines passiv-gütegeschalteten Nd:YAG Lasers durchgeführt. Prozessbegleitend findet eine Aufzeichnung der akustischen Emissionen mit anschließender Analyse durch schnelle FourierTransformation statt. Hierdurch lassen sich der Durchbruch beim Bohren durch ein Material sowie der Materialübergang mehrschichtiger Systeme detektieren. Die akustischen Messungen werden durch Auswertung der Pulsfolge des Lasers mittels einer Fotodiode gestützt. Hierbei zeigt sich eine gute Übereinstimmung der im akustischen Spektrum dominanten Frequenz mit der jeweils im Laserburst auftretenden Pulsfrequenz. Das vorgestellte Verfahren ermöglicht eine Echtzeitüberwachung beim Laserbohren mittels kostengünstiger und einfacher Hardware. Zudem zeichnet es sich im Gegensatz zu bestehenden Verfahren durch eine hohe Robustheit gegen äußere Störeinflüsse aus, da eine frequenzbasierte Auswertung stattfindet.
\end{abstract}

Schlagwörter: Lasermaterialbearbeitung, Laserbohren, Echtzeitüberwachung, Frequenzauswertung

\section{Einleitung}

Bei vielen Anwendungen des Laserbohrens ist eine exakte Charakterisierung der Bohrlocheigenschaften wichtig, um im Sinne einer Qualitätskontrolle die Durchführung einer präzisen und korrekten Bohrung gewährleisten zu können. So ist es beispielsweise bei der Herstellung von Flugzeugturbinen notwendig, einige Tausend feine Bohrlöcher zu fertigen, deren wichtigste Kenngrößen nur geringe Toleranzen aufweisen dürfen [1].

In der industriellen Praxis werden hierbei Verfahren bevorzugt, die eine Echtzeitanalyse während der Laserbearbeitung gestatten. Hierdurch kann auf zeitaufwendige und kostspielige Prüfverfahren durch optische Nachkontrollen, Luftströmungstests am Werkstück oder Stiftchecken der Bohrlöcher verzichtet werden [2]. Auch für den am Institut für Detektionstechnologien verfolgten Ansatz der laserbasierten Probenahme verpackter Gefahrstoffe ist eine schnelle und sichere Echtzeitüberwachung des Bohrvorgangs von entscheidender Bedeutung [3].

Ein indirekter Indikator der Eigenschaften eines lasergebohrten Loches ist die Durchbruchzeit im Vergleich zur gesamten Bearbeitungsdauer, da es nach erfolgtem Durchbruch zu einer Aufweitung des bestehenden Bohrkanals kommt. In der Literatur beschriebene sensorische Verfahren zur Echtzeitüberwachung von Bohrprozessen basieren oft auf einer Auswertung der hierbei auftretenden optischen und akustischen Emissionen [4]. Diese Verfahren sind jedoch insbesondere dann anfällig für äußere Störeinflüsse, wenn lediglich Änderungen von Signalintensitäten detektiert werden.

In der vorliegenden Arbeit wird daher ein neuartiges Verfahren zur Überwachung von Laserbohrprozessen vorgestellt, das eine frequenzbasierte Auswertung durchführt und hierdurch besonders robust gegen äußere Störeinflüsse ist.

\section{Methoden und Materialien}

Für die Durchführung der Perkussionsbohrungen an unterschiedlichen Werkstücken wird ein diodengepumpter und passiv gütegeschalteter Nd:YAG Laser in OszillatorVerstärker-Anordnung verwendet (Wellenlänge 1064 nm, Pulsdauer ca. 3 ns, mittlere Pulsenergie $3 \mathrm{~mJ}$ ). Der Laser wird mit einer 
Repetitionsrate von $25 \mathrm{~Hz}$ im sogenannten Burst-Modus betrieben, bei dem während eines Pumppulses mehrere Einzelpulse emittiert werden. Der exakte Laseraufbau ist in [5] beschrieben. Die Laserstrahlung wird mit einer antireflektiv beschichteten Plankonvexlinse der Brennweite $f=170 \mathrm{~mm}$ auf die Oberfläche des Werkstücks fokussiert. Während des Bohrvorgangs wird der hierbei entstehende Luftschall durch ein ca. $10 \mathrm{~cm}$ vom Werkstück entfernt angebrachtes Elektret-Mikrofon (Bandbreite ca. $25 \mathrm{~Hz}-30 \mathrm{kHz}$ ) aufgezeichnet. Zudem wird mit einer Silizium-Fotodiode die emittierte Pulsfolge am Auskoppelspiegel des Lasers registriert. Beide Sensoren werden mit einer Samplerate von $120 \mathrm{kHz}$ ausgelesen. Zur Detektion des Durchbruchs beim Bohren dient ein hinter dem Werkstück angeordnetes Leistungsmessgerät. Eine Skizze des Experimentalaufbaus ist in Abbildung 1 dargestellt.

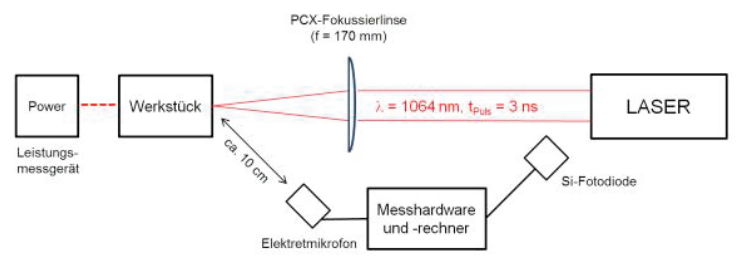

Abbildung 1: Skizze des Experimentalaufbaus mit Strahlführung und Anordnung der Sensoren. Für den Aufbau des verwendeten Nd:YAG Lasers sei auf [5] verwiesen

Untersucht werden verschiedene Metallplättchen (Aluminium, Kupfer, Messing) unterschiedlicher Dicke sowie Materialkombinationen aus Metall und Kunststoff. Mittels schneller Fourier-Transformation (fast fourier transformation, FFT) wird aus dem Audiosignal jedes Laserbursts ein akustisches Frequenzspektrum errechnet. Hierzu wird ein eigens geschriebenes LabView@ - Programm verwendet. Der zur Auswertung gewählte Bereich ist in Abbildung 2 exemplarisch für den mittleren Laserburst dargestellt und wird durch ein Hann-Fenster eingeschränkt. Für die FFTAnalyse werden jeweils 1024 Datenpunkte verwendet, um hieraus ein logarithmisch skaliertes Leistungsspektrum zu berechnen.

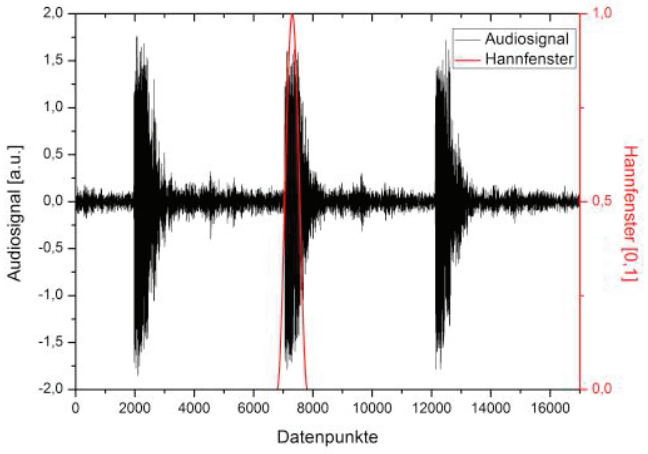

Abbildung 2: Audiosignal dreier Laserbursts beim Bohren in Aluminium. Die Einzelpulse im Burst können durch die akustische Messung nicht aufgelöst werden. Der Bereich für die Berechnung der FFT mit 1024 Datenpunkten wird durch das dargestellte Hann-Fenster eingeschränkt

\section{Ergebnisse}

Abbildung 3 zeigt die Frequenzauswertung eines typischen Bohrvorgangs in einem $0.3 \mathrm{~mm}$ dicken Aluminiumplättchen in Spektrogrammdarstellung. Auf der Ordinate ist der zeitliche Bohrverlauf in Form der Laserbursts dargestellt, wobei 25 Laserbursts einer Bohrdauer von einer Sekunde entsprechen. Die Abszisse zeigt die mittels FFT errechneten Frequenzspektren des aufgezeichneten Luftschallsignals. Die Amplituden der einzelnen Frequenzbänder sind durch eine logarithmische Farbskala von $-65 \mathrm{~dB}$ bis $0 \mathrm{~dB}$ kodiert. Deutlich erkennbar ist eine Änderung und Homogenisierung des Spektrums ab dem 25. Laserburst, was einem Zeitpunkt von einer Sekunde nach Start der Bohrung entspricht. Dieser Zeitpunkt korreliert mit dem Durchbruch des Plättchens, was durch Messung mit dem Leistungsmessgerät festgestellt wird. Ähnliche Beobachtungen ergeben sich bei der Untersuchung von Bohrungen in Kupfer und Messing.

Auch bei Werkstücken aus mehrschichtigen Materialien zeigt sich eine Änderung der akustischen Spektren im zeitlichen Verlauf der Bohrung. Beispielhaft sei hier auf das Spektrogramm einer Polystyrol/Aluminium Probe in Abbildung 4 verwiesen. Auch hier lässt sich eine deutliche Veränderung der Spektren beim Materialübergang sowie beim Durchbruch erkennen. 


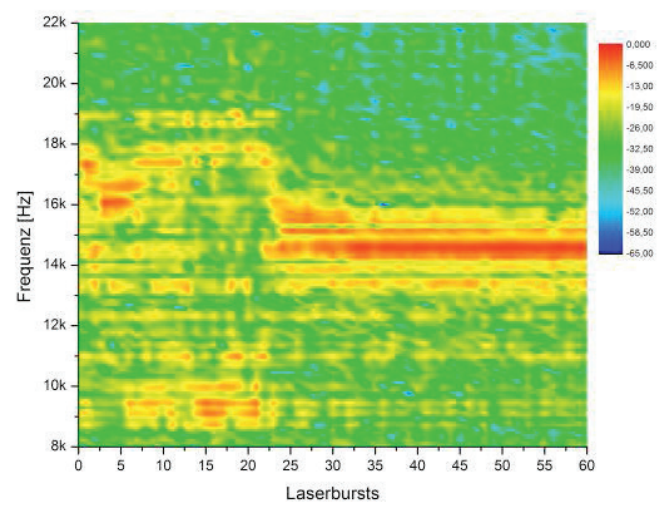

Abbildung 3: Spektrogramm der Bohrung in einem $0,3 \mathrm{~mm}$ dicken Aluminiumplättchen. Es zeigen sich deutliche Änderungen im akustischen Spektrum beim Durchbruch der Bohrung ab dem 25. Laserburst

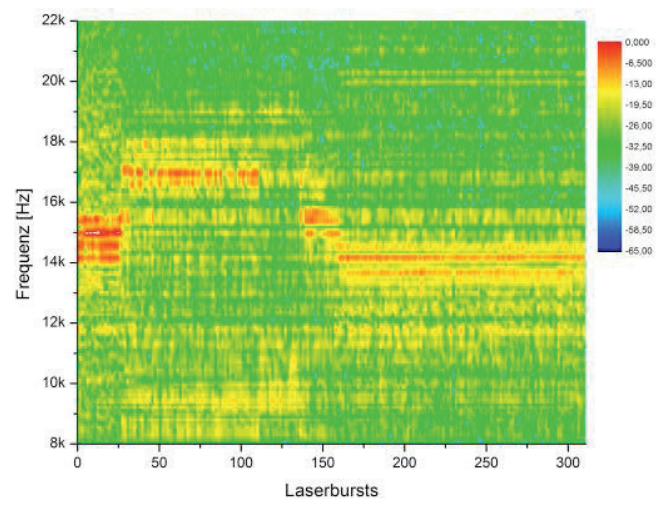

Abbildung 4: Bohrung in einer Materialkombinationsprobe bestehend aus Polystyrol (0,6 $\mathrm{mm})$ und Aluminium $(0,3 \mathrm{~mm})$. Die Änderung des Spektrums ab dem 150. Laserburst korreliert mit dem Durchbruch durch beide Schichten. Ab dem 25. Laserburst zeichnet sich der Übergang zwischen den beiden Materialschichten ab

Aus den errechneten Akustikspektren ist in Abbildung 5 die Frequenz mit der stärksten Amplitude gegen die Laserbursts aufgetragen. Es ergibt sich im Frequenzverlauf eine gute Übereinstimmung mit der Darstellung im Spektrogramm (Abbildung 4). Auch hier zeigt sich eine deutliche Verschiebung der stärksten Frequenz in Korrelation mit dem Durchbruch der Bohrung sowie beim Materialwechsel von Polystyrol auf Aluminium. Die Darstellung wurde zur besseren Visualisierung um einen gleitenden Durchschnitt mit einer Fensterbreite von 10 Datenpunkten ergänzt.

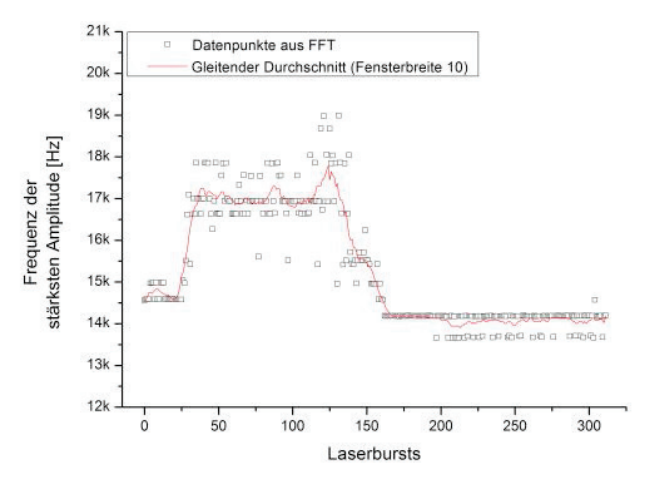

Abbildung 5: Verlauf der Frequenz mit stärkster Amplitude beim Bohren in einer Polystyrol/Aluminium - Probe. Es zeigt sich sowohl ab dem 25. als auch ab dem 150. Laserburst eine deutliche Verschiebung der stärksten Frequenz im Spektrum

Die Auswertung der akustischen Emissionen wird durch die Aufnahmen der Fotodiode am Laserausgang gestützt. Für die Auswertung wurde bei jedem Laserburst separat der Mittelwert der zeitlichen Abstände der Einzelpulse im Burst berechnet und hiervon der Kehrwert gebildet. In Abbildung 6 ist das Ergebnis dieser Auswertung zusammen mit der Hauptfrequenz der akustischen Spektren aufgetragen. Beide Frequenzverläufe wurden mit einem digitalen Bandpass $(10 \mathrm{kHz}-24$ $\mathrm{kHz}$ ) bearbeitet. Es zeigt sich eine große Übereinstimmung beider Frequenzverläufe in der akustischen und optischen Auswertung.

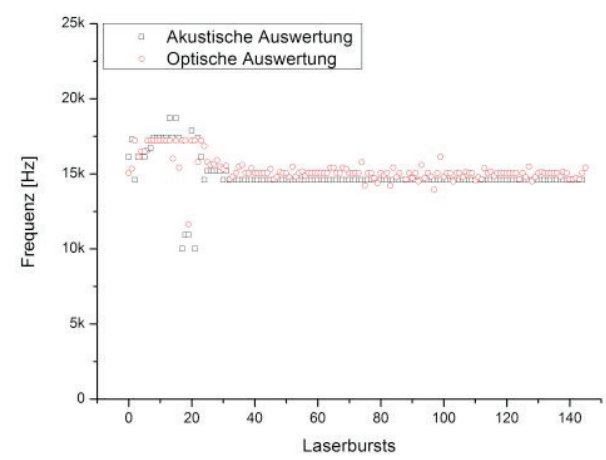

Abbildung 6: Zusammengeführte Darstellung der akustischen und optischen Auswertung für eine Bohrung in Aluminium (0,3 mm). Für die akustische Auswertung ist die Frequenz mit der stärksten Amplitude aufgetragen, für die optische Auswertung wird die mittlere Pulsfrequenz im Laserburst dargestellt 


\section{Diskussion}

Es zeigt sich deutlich, dass durch Analyse des Frequenzspektrums der akustischen Emissionen und durch die Auswertung der Pulsfrequenz im Laserburst eine einfache und echtzeitfähige Überwachung des Bohrvorgangs ermöglicht wird. So lassen sich sowohl Materialübergänge im Werkstück als auch der Durchbruch einer Bohrung pulsgenau detektieren.

Die Messungen der Fotodiode zeigen, dass im akustischen Spektrum die Frequenz der Repetitionsrate des Lasers dominiert, welche sich folglich in Abhängigkeit des Bohrfortschritts geringfügig ändert. Eine Variation des zeitlichen Auftretens der Pulse ist bei Lasern mit passiver Güteschaltung im Gegensatz zu aktiv gütegeschalteten Systemen grundsätzlich möglich und wird als Jitter bezeichnet [6].

Dem beobachteten Effekt zugrunde liegend sind vermutlich Rückstreuungen der vom Werkstück reflektierten Strahlung in den Laser. Das Werkstück und der Laser bilden hierdurch ein System gekoppelter optischer Resonatoren. Dies wird durch die optische Verstärkung der reflektierten Strahlung in der OszillatorVerstärker-Anordnung zusätzlich begünstigt. Unkontrolliert kann dies bis zu einem in der Literatur als "chaotischer Betrieb" beschriebenem Zustand führen [7]. In den hier vorgestellten Untersuchungen lässt sich dieser Effekt jedoch für die sensorische Überwachung des Bohrvorgangs vorteilhaft ausnutzen, zumal die Qualität der Bohrungen durch die geringfügige Änderung der Pulsfrequenz nicht beeinträchtigt wird.

Für die Echtzeitüberwachung wird letztendlich ausgenutzt, dass in Abhängigkeit von Material und Prozessfortschritt unterschiedlich starke Anteile der Laserstrahlung vom Werkstück zurück in den Resonator des Lasers reflektiert werden. Denkbar ist, dass die zurückgekoppelte Strahlung einerseits Photonen für den nächsten Laserpuls injiziert und außerdem für eine teilweise Sättigung des Absorbers im Güteschalter sorgt. Dies führt zu einer Verschiebung der Energieschwelle, die für die Emission des nächsten Einzelpulses überschritten werden muss [8]. Folglich lässt sich eine Verschiebung der relativen zeitlichen Abstände der Einzelpulse im Burst und somit eine Änderung der Pulsfrequenz messen, die sich auch im akustischen Spektrum der Bohrung niederschlägt. So lässt sich zu Beginn des Bohrvorgangs eine Erhöhung der Pulsfrequenz beobachten, da hier noch ein vergleichsweise großer Anteil der Strahlung reflektiert wird.
Die Ergebnisse zeigen, dass mit dem vorgestellten Verfahren eine Detektion des Durchbruchs beim Laserbohren möglich ist. Auch lässt sich der Übergang zwischen unterschiedlichen Materialschichten in Echtzeit verfolgen. Dabei ist das Verfahren messtechnisch besonders kostengünstig und ohne Modifikationen am Laseraufbau realisierbar. Durch die frequenzbasierte Auswertung ergibt sich zudem eine im Gegensatz zu bestehenden Verfahren geringere Anfälligkeit gegenüber äußeren Störeinflüssen.

\section{Literatur}

[1] DUBEY, A.K. ; YADAVA, V.: Laser beam machining - A review. Int. J. of Machine Tools and Manufacture 48 (2008), Nr. 6, S. $609-628$

[2] ORTIZ A.L.Jr. ; SCHNEITER, J.: Method and apparatus for optically/acoustically monitoring laser materials processing. US-Patent 5045669A (1991), General Electric Company

[3] WILD, D. ; THEISS, C. ; KAUL, P. ; HOLL, G.: Robot-based laser drilling system for the detection of concealed threats. AUBE 2014 Proceedings, S. 177-182

[4] DEININGER, C.: Prozessüberwachung in der Lasermaterialbearbeitung. Laser Technik Journal 3 (2006), Nr. 4, S. 29-32

[5] PEUSER, P. ; PLATZ, W. ; HOLL, G.: Miniaturized, high-power diode-pumped, Q-switched Nd:YAG laser oscillatoramplifier. Applied Optics 50 (2011), Nr. 4, S. 399-404

[6] SENNAROGLU, A.: Solid-State Lasers and Applications. CRC Press (2006), S. 29-30

[7] BLIEDTNER, J. ; MÜLLER, H. ; BARZ, A.: Lasermaterialbearbeitung: Grundlagen Verfahren - Anwendungen - Beispiele. Carl Hanser Verlag GmbH \& Co. KG (2013), S. $119-121$

[8] STEINMETZ, A. et al.: Reduction of timing jitter in passively Q-switched microchip lasers using self-injection seeding. Optics Letters 35 (2010), Nr. 17, S. 2885-7

\section{Danksagung}

Wir danken der Firma ExploTech $\mathrm{GmbH}$ für die Bereitstellung des für die durchgeführten Versuche verwendeten Lasersystems. 